\title{
Nuclear cardiology in the UK: do we apply evidence based medicine?
}

\author{
S L Rahman, A D Kelion
}

Heart 2004;90(Suppl V):v37-v40. doi: 10.1136/hrt.2002.005165

? oronary artery disease is one of the greatest health care problems facing the western industrialised nations. Each year in the UK coronary artery disease is responsible for approximately 125000 deaths, 274000 nonfatal myocardial infarctions, and 330000 new presentations of angina. ${ }^{1}$ There have been significant advances in coronary disease treatment over the last few years, with improvements in pharmacological therapy, percutaneous coronary intervention, and bypass surgery. It is therefore increasingly important to identify patients who will benefit from these approaches from the large number of people presenting with chest pain. The cost of identifying this group is putting health care systems under severe financial strain as much as the expense of the treatment itself. The importance of this issue was recognised by the UK government with the publication of its National Service Framework (NSF) for coronary heart disease document in 2000, linked to the provision of significant additional funding. ${ }^{2}$

An extensive body of evidence has accumulated over the last 20 years demonstrating the ability of nuclear cardiology techniques, particularly myocardial perfusion imaging (MPI), to identify and risk stratify patients with coronary artery disease. ${ }^{3}$ Strategies which involve MPI in the investigation of patients with chest pain have been shown to be cost effective as well as clinically effective. ${ }^{4-6}$ MPI is therefore a potentially valuable tool in a resource limited system such as the National Health Service (NHS) in the UK. This article will attempt to define a realistic evidence based role for MPI in UK cardiology and will then review current practice in terms of both the number and quality of investigations performed.

\section{INVESTIGATING THE PATIENT WITH CHEST PAIN}

Chest pain is a very common symptom in the general population but in only a minority of patients does coronary disease turn out to be the cause. A careful history will identify a significant group whose chest pain is clearly non-ischaemic in origin and who require reassurance only. For the remainder, clinical assessment alone is notoriously unreliable at providing a confident diagnosis or prognosis. Coronary angiography remains the definitive investigation for obstructive coronary disease in clinical practice and is required to guide percutaneous or surgical revascularisation where necessary. However, it is expensive and invasive, with a small but definite risk of complications. The investigation of all patients presenting with chest pain by coronary angiography would be clinically effective but prohibitively expensive. This has driven the development of alternative noninvasive investigations to distinguish between high risk patients most likely to benefit from angiography and subsequent revascularisation, and low risk patients who can safely be managed conservatively.

The cheapest and most widely available first line investigation for patients presenting with chest pain is the standard exercise ECG. Although the exercise test can identify clear cut low risk and high risk groups, one third or more of patients emerge with an intermediate risk of coronary disease or cardiac events, which does not advance their management. ${ }^{7}$ There are three groups in which the exercise ECG is particularly unhelpful: (1) patients unable to exercise to the target heart rate; (2) patients with an abnormal resting ECG (particularly left bundle branch block), which hampers the interpretation of changes during exercise; (3) women, for whom the test is of reduced positive and negative predictive value compared with men because of the generally lower pretest probability of coronary disease, the greater chance of failing to achieve the target heart rate, and the higher rate of false positive ST depression. ${ }^{8}{ }^{9}$ The temptation is often to err on the side of caution and refer such patients for coronary angiography, accepting that a high proportion will have normal coronary arteries.

MPI is of proven diagnostic and prognostic value in all groups of patients, including those for whom the exercise ECG is unhelpful. It has an excellent safety profile, and the small risk of complications is related to stress testing (risk of death 1 in 20000 ). For the prediction of coronary stenoses, MPI has a sensitivity and normalcy rate of approximately $90 \% .{ }^{10}$ This is independent of the age and sex of the population studied, the method of stress used, and the choice of thallium or technetium labelled tracer. ${ }^{11-13}$ More importantly, MPI provides robust prognostic information which is independent even of the result of coronary angiography. ${ }^{14}$ A normal MPI study predicts an annual rate of cardiac death or non-fatal myocardial infarction of less than $1 \%$ over the following few years, similar to that for an unselected population not known to have coronary disease. ${ }^{15}$ Even patients with other markers of poor outcome, including ST segment depression during exercise or significant coronary artery disease on angiography, have a good prognosis if MPI is normal. ${ }^{16}{ }^{17}$ In contrast, severe and extensive perfusion defects predict an adverse prognosis. ${ }^{18}$ Although it is a more expensive investigation than the exercise ECG, MPI used selectively but routinely in the management of patients with chest pain would be expected to reduce the number of unnecessary normal coronary angiograms, without detriment to patient outcome.

Two retrospective studies have looked directly at the cost effectiveness of MPI compared with other approaches to the management of chest pain. ${ }^{56}$ The American economics of non-invasive diagnosis (END) study compared 5423 patients investigated directly by coronary angiography with 5826 patients investigated by MPI as a first line test. Thirty four per cent of the MPI patients subsequently underwent angiography. The rates of cardiac events over three years were comparable between the two strategies at every level of pretest clinical risk. However, the cost of care per patient over the same period was $30-40 \%$ lower for those undergoing MPI as the first line investigation.

In the European economics of myocardial perfusion imaging in Europe (EMPIRE) study, there was no difference in outcome over two years between a strategy which relied on the exercise ECG alone, followed by angiography as

Abbreviations: BCS, British Cardiac Society; BNCS, British Nuclear Cardiology Society; EMPIRE, economics of myocardial perfusion imaging in Europe; END, economics of non-invasive diagnosis; MPI, myocardial perfusion imaging; NSF, National Service Framework; SPECT, single photon emission computed tomography 
appropriate (146 patients), compared with one which employed the exercise ECG, followed by MPI, followed by angiography as appropriate (131 patients). However, the management cost per patient over the same period was lower using the MPI strategy by $14 \%$ for those with coronary disease, and by $33 \%$ for those without coronary disease. Thus, the available evidence suggests that the routine use of MPI in the investigation of chest pain is more cost effective than over-reliance on the exercise ECG or direct coronary angiography, with no difference in prognostic outcome.

It must be conceded that both EMPIRE and END were performed retrospectively and a randomised prospective study of management approaches in chest pain, with special emphasis on cost implications, is required to define the precise economic value of MPI.

MPI provides more accurate diagnostic and prognostic information than the exercise ECG, but is more expensive and less accessible. For many patients presenting with chest pain, the exercise ECG is all that is required to reassure those at low risk and select those at high risk for angiography. However, on both clinical and economic grounds, patients presenting with chest pain in who the exercise ECG has been, or is likely to be, unhelpful should seldom be sent for coronary angiography without first being assessed with MPI. This is the current position of the British Cardiac Society (BCS), which in its 1999 guidelines on the investigation and management of stable angina recommended the routine use of MPI rather than the exercise ECG in patients unable to exercise, those with an abnormal resting ECG, and all women (fig 1). ${ }^{19}$

\section{LEVEL OF ACTIVITY OF MPI IN THE UK}

The NSF document on coronary disease has defined a pivotal role for rapid access chest pain clinics in the assessment of patients presenting with recent onset chest pain, although ironically it makes almost no mention of MPI. ${ }^{2}$ The decision to offer coronary angiography to patients attending such clinics is typically based solely on the results of clinical assessment and the exercise ECG, in spite of the BCS guidelines. The experience of a busy chest pain clinic in Southampton between 1998 and 2000 illustrates the problems with this approach and is likely to be typical. ${ }^{20}$ The clinic was attended by 1522 patients with recent onset chest pain-921 were men and 601 were women. Following the exercise ECG, only 3\% of men and $9 \%$ of women were investigated with MPI. Women were less likely to undergo coronary angiography than men $(26 \% v 36 \%)$, but of those who did, a considerably higher proportion were found to have normal coronary arteries (56\% v 16\%). As expected from the literature, the exercise ECG was an unreliable way of selecting women for coronary angiography and the more frequent deployment of MPI would have allowed a more focused use of coronary angiography.

Anecdotal reports such as that from Southampton indicate that MPI is not being used in the routine management of patients with chest pain in the majority of hospitals in the UK. However, if the situation is to be improved it is important to know how the current level of activity compares with a target level. To obtain such data, every few years the British Nuclear Cardiology Society (BNCS) conducts a survey of nuclear cardiology practice in the UK by writing to all identifiable nuclear medicine departments. While the 1988 , 1994, and 1997 surveys have been published, the 2000 survey has only recently been completed though preliminary data are available. ${ }^{21-23}$ The calculation of accurate national rates of MPI from each survey is complicated by the inevitable submaximal response rate to any questionnaire and assumptions must be made about activity in non-responding centres. The MPI rates/1000 population/year in 1988, 1994, 1997, and
2000 are shown in fig 2 . There has been a progressive increase in activity over the 12 years covered by the surveys and the most recent value for 2000, calculated using the most optimistic assumption, is $1.20 / 1000 /$ year.

Compared with the rest of western Europe and the USA, the activity level for MPI in the UK is very low despite one of the highest standardised mortality rates for coronary disease in the world (fig 3).22 In 1994, the European Union average number of MPI investigations was 2.2/1000/year compared with just $0.56 / 1000 /$ year in the UK. The rate in the USA in 1993 was more than 10/1000/year, although differences in the organisation of health care on either side of the Atlantic make direct comparisons difficult. In 1994 the BCS accepted that greater numbers of MPI studies needed to be performed in the UK and set a somewhat arbitrary target of 2.2/1000/ year, the then European average. ${ }^{24}$ It is clear from the 2000 survey that eight years later this target has yet to be achieved and it is likely that since 1994 the European average, and hence the target, has risen substantially.

The 1994 target for MPI activity, set by the BCS, of 2.2/ $1000 /$ year is conservative in relation to targets for other cardiac procedures, as set down in the NSF document in $2000 .^{2}$ It was suggested that there should be 750 coronary artery bypass operations and 750 percutaneous coronary interventions per million population per year. Assuming a rate of two coronary angiograms per revascularisation, this implies a target of three angiograms/1000/year. If the BCS guidelines were applied, more MPIs ought to be performed than coronary angiograms. In fact, the data from the 2000 survey indicate a current ratio of only $0.6: 1$ in centres able to perform both MPI and coronary angiography. Applying an arbitrary, but probably conservative, ratio of $2: 1$, six MPIs/ 1000/year would be required to be consistent with the NSF targets, rather higher than the BCS target from 1994 and substantially higher than the actual level of activity in 2000.

\section{QUALITY OF MPI IN THE UK}

The BNCS surveys have consistently shown that the numbers of MPI studies performed in the UK are significantly lower than would be expected, whether in relation to the activity in other countries or to the number of other cardiac investigations performed. They have also provided an insight into the quality of the studies performed. There is a wide range of acceptable and evidence based practice in many aspects of MPI-for example, in the choice of method of stress and radiopharmaceutical. ${ }^{12}{ }^{13}$ In contrast, there is now general agreement on some important aspects of image acquisition. Single photon emission computed tomography (SPECT) imaging is more accurate for the detection of coronary disease than planar imaging, particularly in patients with relatively mild or single vessel disease. ${ }^{25} 26$ This has been generally accepted in the UK, and in the 2000 survey only $12 \%$ of studies were still being performed using planar acquisitions; these were concentrated in a handful of centres.

Gating of SPECT acquisitions is now well established as a valuable method of identifying attenuation artefacts and for providing important prognostic information about left ventricular function. ${ }^{27}{ }^{28}$ It is therefore disappointing that in 2000 only $29 \%$ of centres were performing any gated SPECT and that overall only $22 \%$ of MPI studies involved gating. This was despite the fact that $59 \%$ of studies employed a technetium-99m based tracer and were therefore candidates for gating (gating of thallium-201 studies is not practicable in the UK because of the limited dosage permitted).

\section{FACTORS LIMITING MPI ACTIVITY IN THE UK}

The shortfall in MPI activity in the UK probably arises from a vicious circle of low perceived demand and inadequate supply. In all but three centres MPI is performed in general 


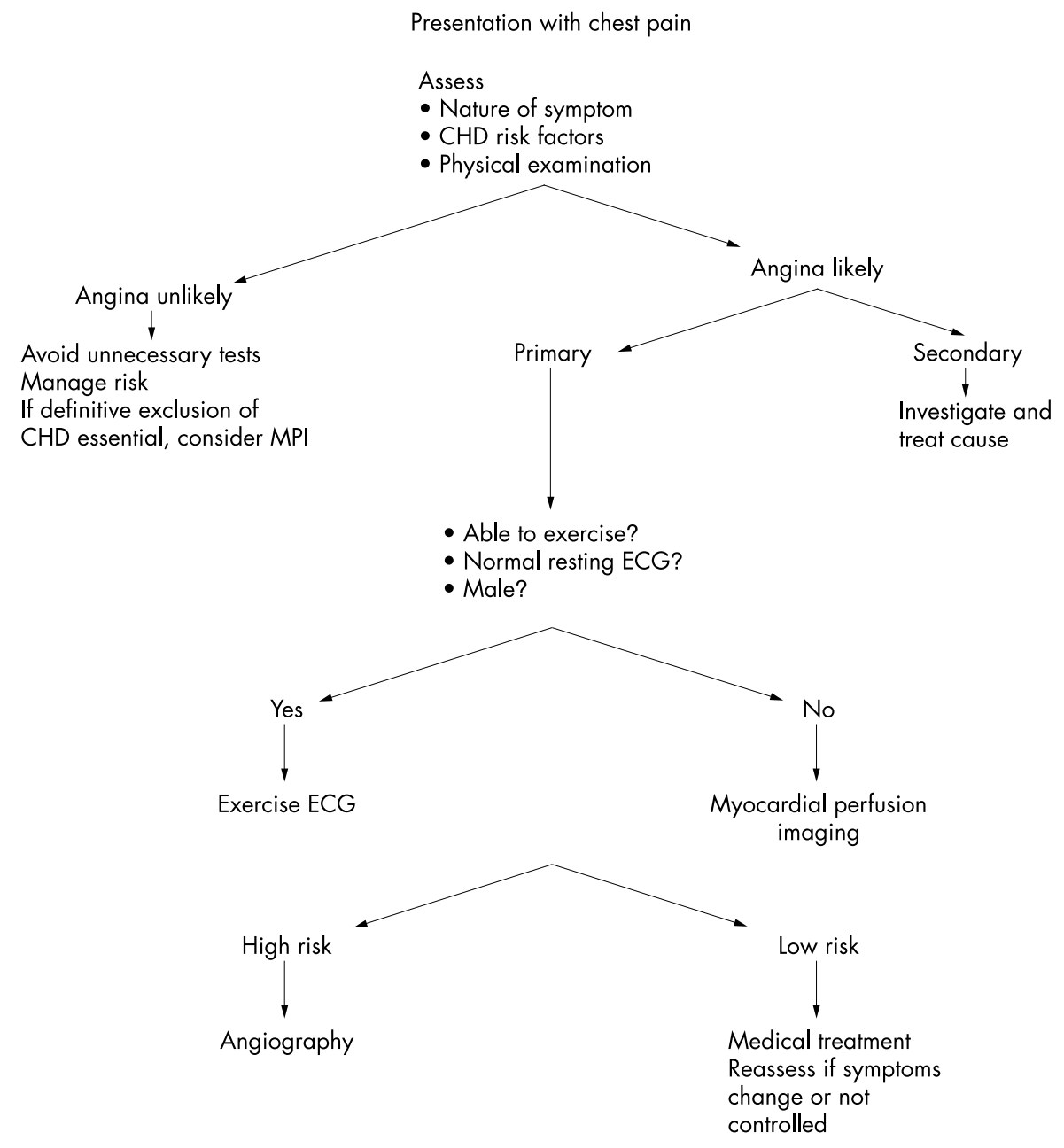

Figure 1 British Cardiac Society guidelines on the investigation and management of stable angina. ${ }^{19} \mathrm{CHD}$, coronary heart disease.

nuclear medicine departments often outside the direct experience and supervision of referring cardiologists. This is in notable contrast to other cardiac investigations such as echocardiography and angiography. In the 2000 survey, a cardiologist was routinely involved in the reporting of MPI studies in fewer than half of the centres $(40 \%)$. Given the subtleties in the modern management of coronary disease, technically accurate reports written by nuclear physicians and radiologists may fail to address important issues in a

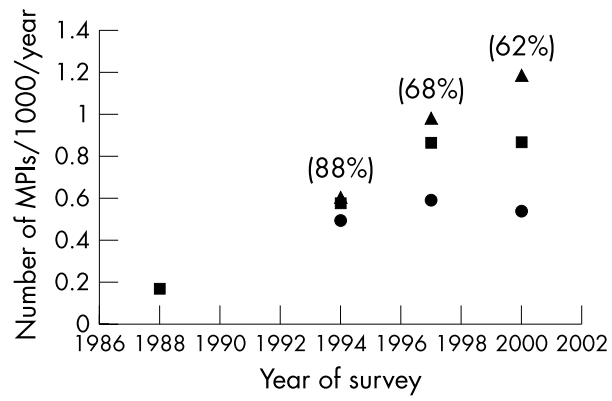

Figure 2 Numbers of MPI studies/1000 population/year from the four British Nuclear Cardiology Society surveys. ${ }^{21-23}$ Response rates for each survey are shown in brackets. Values were calculated using three different assumptions for activity rates in non-responding centres: (1) non-responding centres performed no MPIs (circles); (2) non-responding centres performed the same mean number of MPIs per centre as responding centres (squares); (3) non-responding centres performed the same mean number of MPIs per centre as responding centres which performed MPI (triangles). particular case or may frankly be misleading to a referring cardiologist with a rudimentary understanding of MPI. For example, over-reporting of straightforward attenuation artefacts as possible perfusion defects can lead to a series of normal coronary angiograms, causing an initially sceptical cardiologist to lose confidence in the technique entirely and stop referring patients.

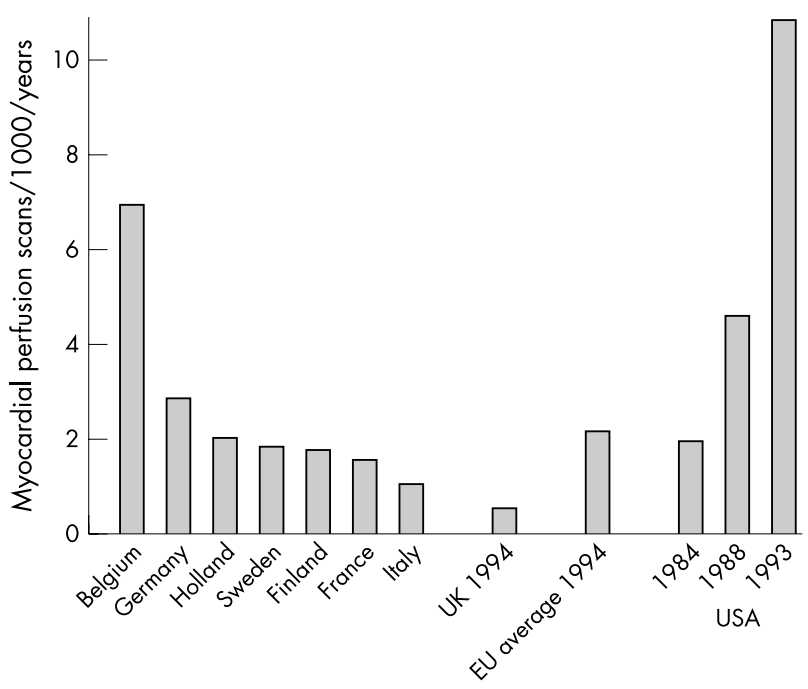

Figure 3 Number of MPI studies/1000 population/year in 1994 in the UK, compared with other European countries and trends in the USA. ${ }^{22}$ 
The BNCS asked an expert panel to audit the quality of 90 MPI acquisitions and reports from 18 UK centres. ${ }^{29}$ Studies were felt to be technically adequate in the vast majority of cases. However, the text of the report was found to be inadequate for one third of the studies: half were felt to be factually incorrect and half were essentially correct but misleading because of poor wording. A MPI study is only as valuable as the report, however meticulously it has been performed. There is clearly a need in the UK for cardiologists to become more involved in the reporting of MPI studies and for nuclear physicians and radiologists to have a good understanding of the modern management of patients with coronary disease.

The lack of ownership of MPI felt by cardiologists and the resulting low demand is compounded by a lack of capacity. The mean waiting time for a routine MPI study in the 2000 survey was 20 weeks, a level at which direct cardiac catheterisation may become a more efficient way of investigating potentially at-risk patients. The main reasons limiting throughput and causing the long waiting times were limited gamma camera time and insufficient staff to supervise stress tests. Until cardiologists become more involved and nuclear cardiology services are adequately resourced, the argument that MPI is clinically valuable may remain largely academic in many hospitals.

\section{CONCLUSION}

There is now a large body of evidence to suggest that MPI is a clinically effective and cost effective tool in the diagnosis and management of patients with suspected or documented coronary disease. Despite this, the level of activity in the UK remains depressingly low, whether in relation to other cardiac investigations or in relation to practice in comparable industrialised nations. In addition, well validated innovations such as gated SPECT have been slow to establish themselves in routine clinical practice. The focus on cardiac catheterisation by the NSF document, the lack of ownership of MPI felt by many cardiologists, and inadequate resources leading to unacceptably long waiting times in many centres, may all be contributing factors. The argument that MPI is a clinically effective tool in the diagnosis and management of coronary disease remains irrelevant in many hospitals which have no access to a reliable nuclear cardiology service. As the cost of managing coronary disease in the UK becomes ever larger, it is to be hoped that the argument that MPI is highly cost effective will become more persuasive and lead to an expansion of nuclear cardiology services.

\section{Authors' affiliations \\ S L Rahman, Department of Cardiology, St Thomas's Hospital, London, UK}

A D Kelion, Department of Cardiology, Harefield Hospital, London, UK

Correspondence to: Dr Andrew D Kelion, Nuclear Medicine Department, Harefield Hospital, Hill End Road, Harefield, Middlesex UB9 6JH, UK; a.kelion@rbh.nthames.nhs.uk

\section{REFERENCES}

1 British Heart Foundation Health Promotion Research Group. Coronary heart disease statistics 1999. Department of Public Health, University of Oxford. www.dphpc.ox.ac.uk.

2 Department of Health. National service framework for coronary heart disease. London: Department of Health, 2000.
3 American College of Cardiology/American Heart Association. ACC/AHA task force report. Guidelines for clinical use of cardiac radionuclide imaging J Am Coll Cardiol 1995;25:521-47.

4 Patterson RE, Eisner RL, Horowitz SF. Comparison of cost-effectiveness and utility of exercise ECG, single photon emission computed tomography, positron emission tomography, and coronary angiography for diagnosis of coronary artery disease. Circulation 1995;91:54-65.

5 Underwood SR, Godman B, Salyani S, et al. Economics of myocardial perfusion imaging in Europe-the EMPIRE study. Eur Heart $J$ 1999;20:157-66.

6 Shaw LJ, Hachamovitch R, Berman DS, et al. The economic consequences of available diagnostic and prognostic strategies for the evaluation of stable angina patients: An observational assessment of the value of precatheterization ischemia. J Am Coll Cardiol 1999:33:661-9.

7 Mark DB, Shaw L, Harrell FE, et al. Prognostic value of a treadmill exercise score in outpatients with suspected coronary artery disease. N Engl J Med $1991 ; 325: 849-53$

8 Whinnery JE, Froelicher VF, Stuart AJ. The electrocardiographic response to maximal treadmill exercise in asymptomatic men with left bundle branch block. Am Heart J 1997;94:316-24.

9 Morise AP, Diamond GA. Comparison of the sensitivity and specificity of exercise electrocardiography in biased and unbiased populations of men and women. Am Heart J 1995; 130:741-7.

10 Mahmarian JJ, Verani MS. Exercise thallium-201 perfusion scintigraphy in the assessment of coronary artery disease. Am J Cardiol 1991;67:2-11D.

11 Hachamovitch $\mathrm{R}$, Berman DS, Kiat $\mathrm{H}$, et al. Effective risk stratification using exercise myocardial perfusion SPECT in women: gender related differences in prognostic nuclear testing. J Am Coll Cardiol 1996;28:34-44.

12 Gupta NC, Esterbrooks DJ, Hilleman DE, et al. Comparison of adenosine and exercise thallium-201 single-photon emission computed tomography (SPECT) myocardial perfusion imaging. J Am Coll Cardiol 1992; 19:248-57.

13 Sridhara BS, Braat S, Rigo P, et al. Comparison of myocardial perfusion imaging with ${ }^{99 \mathrm{mT}} \mathrm{c}$-tetrofosmin versus ${ }^{201} \mathrm{Tl}$ in coronary artery disease. Am J Cardiol 1993;72:1015-9.

14 Marie PY, Danchin N, Durand JF, et al. Long-term prediction of major ischemic events by exercise thallium-201 single photon emission computed tomography. Incremental prognostic value compared with clinical, exercise testing, catheterization and radionuclide angiographic data. J Am Coll Cardiol 1995;26:879-86

15 Iskander S, Iskandrian AE. Risk assessment using single-photon emission computed tomographic technetium-99 m sestamibi imaging. J Am Coll Cardiol 1998;32:57-62.

16 Vanzetto G, Ormezzano O, Fagret D, et al. Long-term additive prognostic value of thallium-201 perfusion imaging over clinical and exercise stress test in low to intermediate risk patients: Study in 1137 patients with 6-year follow-up. Circulation 1999;100:1521-7.

17 Fattah AA, Kamal AM, Pancholy S, et al. Prognostic implications of normal exercise tomographic thallium images in patients with angiographic evidence of significant coronary artery disease. Am J Cardiol 1994;74:769-71.

18 Machecourt J, Longere P, Fagret D, et al. Prognostic value of thallium-201 single photon emission computed tomographic myocardial perfusion imaging according to extent of myocardial defect. J Am Coll Cardiol 1994;23:1096-106.

19 De Bono D. Investigation and management of stable angina: revised guidelines 1998. Heart 1999;81:546-58.

20 Wong Y, Rodwell A, Dawkins S, et al. Sex differences in investigation results and treatment in subjects referred for investigation of chest pain. Heart $2001 ; 85: 149-52$

21 Underwood R, Gibson C, Tweddel A, et al. A survey of nuclear cardiological practice in Great Britain. The British Nuclear Cardiology Group. Br Heart J 1992;67:273-7.

22 Pennell DJ, Prvulovich E, Tweddel A, et al. Nuclear Cardiology in the UK: British Nuclear Cardiology Society Survey 1994. Nucl Med Comm 1998; 19:305-13.

23 Prvulovich E, Metcalfe MJ. Nuclear cardiology in the UK: Activity and practice 1997. Eur J Nucl Med 2002;29:553-8.

24 British Cardiac Society. Cardiology in the district general hospital. A report of a working group of the British Cardiac Society. Br Heart J 1994;72:303-8.

25 Fintel DJ, Links JM, Brinker JA, et al. Improved diagnostic performance of exercise thallium-201 single photon emission computed tomography over planar imaging in the diagnosis of coronary artery disease: a receiver operating characteristic analysis. J Am Coll Cardiol 1989;13:600-12.

26 Hacot JP, Bojovic M, Delonca J, et al. Comparison of planar imaging and single-photon emission computed tomography for the detection and localization of coronary artery disease. Int J Card lmaging 1993;9:113-9.

27 DePuey EG, Rozanski A. Using gated technetium-99 m-sestamibi SPECT to characterize fixed myocardial defects as infarct or artifact. J Nucl Med 1995;36:952-5.

28 Sharir T, Germano G, Kavanagh PB, et al. Incremental prognostic value of post-stress left ventricular ejection fraction and volume by gated myocardial perfusion single photon emission computed tomography. Circulation 1999; 100:1035-42.

29 Prvulovich EM, Jarritt PH, Vivian GC, et al. Quality assurance in myocardial perfusion tomography: A collaborative BNCS/BNMS audit programme. Nud Med Comm 1998;19:831-8. 